Proceedings of the 1993 IEEE/RSJ International Conference on Intelligent Robots and Systems Yokohama, Japan July 26-30, 1993

\title{
Estimating Fractal Dimension of Natural Terrain from Irregularly Spaced Data
}

Kenichi Arakawa

NTT Human Interface Labs

3-9-11 Midori-cho Musashino-shi

Tokyo 180 Japan

email: arakawa@nttarm.ntt.jp
Eric Krotkov

The Robotics Institute

Carnegie Mellon University

Pittsburgh, PA 15213 USA

email: Eric.Krotkov@cs.cmu.edu
Abstract

Autonomous mobile robots for exploring natural environments must be capable of terrain whodeling, including texcin roughness. estimation Even if the robot has sensor that can sernse depth. Whis information generally insufficient for accurate terrain mod-

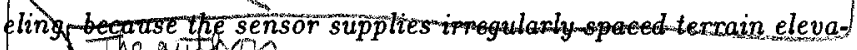
the authers This poropos propos a method to estimate terrain roughness directly from the depth information.

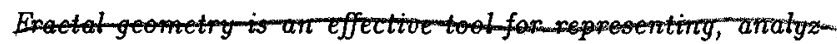
ing and modeting rugged natural shapes-The - Thricept of dimension, whict is retated to the perceived roughness of shapes, ptays pivotrote inthe geometr. Inthis paper, we faltimate the fractal dimension of terrain using the fragtal Brownian function approach. For experiments with real data, fxtend the approach to accommodate irregularly sampled elevation data supplied by a scanning laser rangefinder. Applying this extended method to noisy range imagery of natural terrain (sand and rocks), find that the resulting estimates of fractal dimension correlate closely to human perception of the roughness of the terrain, showing that the fractal dimension of the sensed point set is a practical and effective measure of the roughness of natural terrain.

\section{Introduction}

Robots that operate in natural environments require capabilities to model natural shapes and to analyze relatively unstructured surfaces and objects. One example of such a robot that has motivated our work is the Ambler (Figure 1), a prototype planetary rover designed to operate autonomously in Mars-like terrain [3] Modeling natural shapes is a challenging task for robot vision, and many problems in the analysis and the model construction of natural shapes remain unsolved. One reason is that the familiar Euclidean geometry of regular shapes, such as surfaces of revolution, does not capture well the irregular and less structured shapes found in nature, such as a boulder field, or surf washing onto a beach.

Mandelbrot [9] proposed fractals as a family of mathematical functions to describe natural phenomena such as coastlines. Figure 2 illustrates three fractal patterns with different fractal

This research was sponsored by NASA under Grant NAGW-1175. 0-7803-0823-9/93/\$3.00 (C) 1993 IEEE

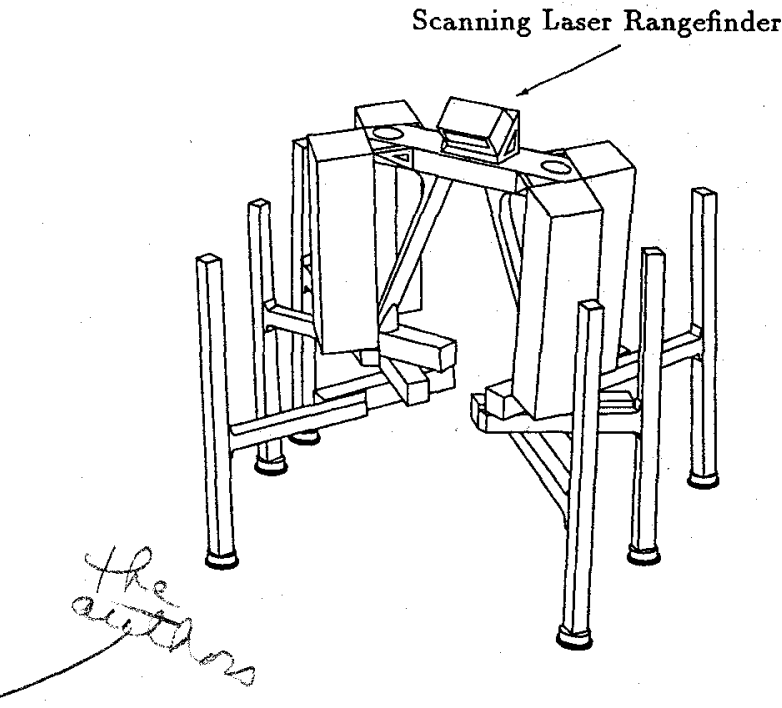

Figure 1: Ambler: a prototype of a rover for exploring natural terrain

The Ambler is equipped with a scanning laser rangefinder called the Perceptron.

dimensions. Clearly, fractal dimension bears a strong relation to perceived roughness of the patterns.

Since Mandelbrot introduced them, fractal sets and functions have been found to describe many environmental properties, and have received a great deal of attention from scientists, artists, and others. Researchers in computer graphics and image understanding have applied fractal theory to a variety of problems. In computer graphics, fractal theory has been used to synthesize models of complex natural objects such as trees, mountain ranges, and clouds [12]. The rendered images and models exhibit a high level of realism compared to previous efforts. In image understanding, fractal theory has been employed to analyze projections of natural scenes for such purposes as modeling the shapes of natural objects and interpolating natural surfaces.

For modeling shapes of natural objects, Pentland [10] presents a method to estimate fractal dimension from the "second-order statistics" of image intensities, and shows that measurements of fractal dimension may be used to measure perspective gradient, thus providing an independent check on estimates of surface orientation derived from analysis of foreshortening. Kube and Pent- 


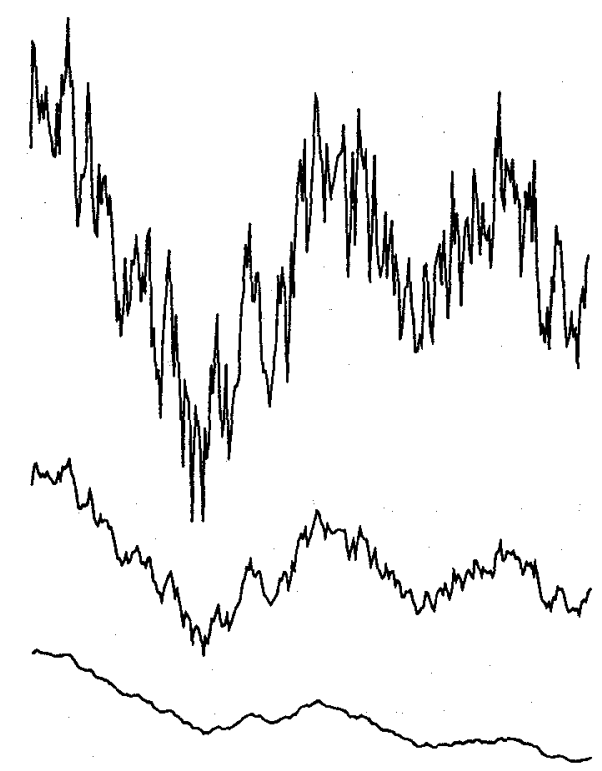

Figure 2: Synthesized one-dimensional fractal patterns The figure shows three fractal patterns exhibiting fractional Brownian motion. For the upper pattern, the fractal dimension is $D=1.8$. For the middle, $D=1.5$, and for the lower, $D=1.2$.

land [5] report that, given certain assumptions (Lambertian reflectance, modest surface slopes, and the absence of occlusions and self-shadowing), a fractal surface with power spectrum proportional to $f^{-\beta}$ produces an image with power spectrum proportional to $f^{2-\beta}$.

For interpolating natural surfaces, Yokoya et al. [15] adopt a recursive midpoint displacement scheme using four neighbors to interpolate natural surfaces. They compute two features-a self-similarity parameter and the standard deviation of the distribution function - and use them for stochastic interpolation in order to preserve the statistical characteristics of the true surface. Szeliski [11] shows that surfaces interpolated using regularization techniques are fractal, and applies a blend of the thin plate and membrane models to generate constrained surfaces with a given fractal dimension. Recently we proposed an extended method of the fractal interpolation based on regularization and showed much more realistic results of elevation maps from depth information sensed by a rangefinder [2].

The work on modeling natural shapes and interpolating natural surfaces represents significant advances. In this paper, we extend the work on estimating fractal dimension to patterns of natural terrain acquired by a laser rangefinder [7]. The sensor supplies fairly noisy depth data, and the observed points are not spaced regularly. In Section 2, we review the fractal Brownian function approach proposed for estimating fractal dimension from digitized data and extend it to handle range data. In Section 3 , using the extension, we present experimental results of estimating fractal dimension of natural terrain from real range images. Finally, we discuss the results, and conclude that the estimated fractal dimension can be used as a reasonable measure of the roughness of natural terrain, because it corresponds to the perceived roughness.

\section{Fractal Brownian Function Approach}

One class of fractals is created by a process called fractional Brow* nian motion. The fractal Brownian function approach applies to this class of fractal patterns. In this section, we explain the mathematical concepts and discuss a method proposed for using them to estimate fractal dimension from range data.

\subsection{Definitions}

Fractional Brownian motion $B_{H}(t)$ is a stochastic process that generalizes classical Brownian motion. It is defined as follows.

1. $B_{H}(0)=$ constant.

2. For constant $C$,

$$
\begin{aligned}
& B_{H}(t)-B_{H}(0)= \\
& C\left[\int_{-\infty}^{0}\left\{(t-s)^{H-1 / 2}-(-s)^{H-1 / 2}\right\} d B(s)\right. \\
& \left.\quad+\int_{0}^{t}(t-s)^{H-1 / 2} d B(s)\right] .
\end{aligned}
$$

A trace of $B_{H}(t)$ exhibits a statistical scaling behavior. If the scale $t$ is changed by the factor $r$, then the increments $\Delta B_{H}(t)$ change by a factor $r^{H}$.

$$
\Delta B_{H}(r t) \propto r^{H} \Delta B_{H}(t) .
$$

If $H=1 / 2$, then $B_{H}(t)$ represents classical Brownian motion. Traces of fractional Brownian motion are statistically self-affine, therefore, they belong to one class of fractals.

Pentland [10] defined a fractal Brownian function as an extension of statistical self-affinity that characterizes self-affine processes, including fractional Brownian motion. A random function $f(t)$ is a fractal Brownian function if, for all $t$ and $\Delta t$, there exists $H$ in

$$
\operatorname{Pr}\left\{\frac{f(t+\Delta t)-f(t)}{\Delta t^{H}}<x\right\}=g(x)
$$

which is independent of $\Delta t$, where $g(x)$ is a cumulative distribution function. In this definition, $\Delta f_{\Delta t}=f(t+\Delta t)-f(t)$ is statistically self-affine, and $H$ is a self-affinity parameter, related to the fractal dimension $D$ of $f(t)$ by $D=2-H$. If $g(x)$ is a zero-mean Gaussian with unit variance, then $f(t)$ represents fractional Brownian motion $B_{H}(t)$.

Interpreting $t$ as a vector quantity $t$ extends this definition to higher topological dimensions. In this case, the $\Delta t$ appearing in the denominator of (1) must be rewritten as the norm $\|\Delta t\|$. If $f(\mathbf{t})$ is a pattern whose domain $\mathbf{t}$ is $E$-dimensional, then $D=$ $E+1-H[13]$. For instance, if we analyze fractal dimension of natural terrain, we can express the terrain as an elevation map $f(t)$ on a horizontal plane $t=(x, y)$, and the fractal dimension can be estimated by $D=3-H$.

Pentland [10] proves that under certain conditions (constant illumination, constant albedo, and a Lambertian surface reflectance function), a three-dimensional surface with a spatially 
isotropic fractal Brownian shape produces an image (i) whose intensity surface is fractal Brownian, and (ii) whose fractal dimension is identical to that of the components of the surface normal. He also shows that the definition of a fractal Brownian function on intensity $I(t)$-instead of $f(t)$ in (1)-can be rewritten as

$$
E\left(\Delta I_{\|\mid \Delta t\|)}\right)\|\Delta t\|^{-H}=E\left(\Delta I_{\|\Delta t\|=1}\right),
$$

where $E\left(\Delta I_{\|\Delta t\|}\right)$ is the expected value of the change in intensity $I(\mathbf{t})$ over distance $\|\Delta \mathbf{t}\|$.

Yokoya $[14,15]$ assumed that intensity in images is distributed by a fractal Brownian function following Pentland's assumption, and additionally that $g(x) \sim N\left(0, \sigma^{2}\right)$. He computed the expected value of the change of intensity $E\left(\Delta I_{\| \Delta t||}\right)$ from images of a planetary surface and found the points $\left(\log \|\Delta t\|, \log E\left(\Delta I_{|| \Delta t||}\right)\right)$ to lie on straight lines. ¿From these lines in log-log space, he estimated each of the slopes $H$, which is

$$
H=\frac{\partial \log E\left(\Delta I_{\|\Delta t\|}\right)}{\partial \log \|\Delta t\|} .
$$

He also estimated fractal dimension from coarse but regularly sampled terrain maps using the same method.

This method is reasonably robust against noisy data, because it uses statistics computed from a large number of data points, and tolerates zero-mean normally distributed sensor noise, because the method implicitly performs an averaging operation. Further discussion regarding the robustness of the method can be found in $[1]$.

\subsection{Extension to Range Images}

We apply the fractal Brownian function approach proposed by Yokoya to range images acquired with a scanning laser rangefinder manufactured by Perceptron, which is located on the sholder of the Ambler (See Figure 1). The data is elevation rather than image intensity, so we change our notation to use $z(d)$ (with $\mathbf{d}=(x, y))$ instead of $I(\mathbf{t})$.

The proposed method for estimating fractal dimension requires regularly sampled data. However, the Perceptron sensor acquires range images with respect to a spherical-polar coordinate system. Equal sampling intervals in this coordinate system become unequal and irregular when mapped into a Cartesian system, as illustrated in Figure 3. Thus, the Perceptron data points are not equally spaced when expressed in Cartesian coordinates, so we have to extend the fractal Brownian function approach to accommodate this irregularity.

\section{Procedure}

The procedure for estimating the fractal dimension from an input Perceptron range image is stated in the five following steps.

1. Delete corrupted pixels.

Known problems with the Perceptron sensor, such as internal reflections and vignette effects, cause some range image pixels to have incorrect or invalid depth values. We remove these pixels by thresholding specified image regions [7].

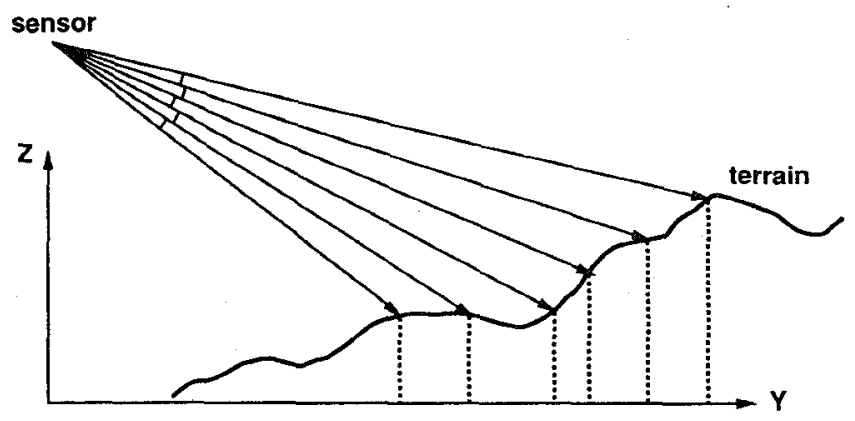

Figure 3: Irregular sampling intervals

The Perceptron rangefinder uses a spherical-polar coordinate system. The equal angle sampling intervals shown are not uniform in a Cartesian system.

2. Transform range image measurements from sensor-centered polar coordinates into sensor-centered Cartesian coordinates.

Given the polar coordinates $\left(r, c, p_{r, c}\right)$ of a pixel located in the row $r$ and column $c$ of the range image, we find coordinates $(x, y, z)$ by

$$
x=\rho \sin \theta, y=\rho \cos \phi \sin \theta, \quad z=\rho \sin \phi \sin \theta,
$$

$\rho=q p_{r, c}+o, \phi=\frac{r \phi_{\text {fov }}}{N_{\text {rows }}-1}+\phi_{\text {start }}, \theta=\frac{c \theta_{\text {fov }}}{N_{\text {rows }}-1}+\theta_{\text {start }}$, where $q$ and $o$ denote the quantization error (meters per greylevel) and standoff distance (meters) of the sensor, $\phi_{\text {fov }}$ and $\phi_{\text {start }}$ denote the horizontal field of view and the horizontal start angle, $\theta_{\text {fou }}$ and $\theta_{\text {start }}$ denote the vertical field of view and the vertical start angle, and $N_{\text {rows }}$ and $N_{\text {cols }}$ denote the numbers of rows and columns in the range image.

3. Compute statistics of $\left|z_{x+d x, y+d y}-z_{x, y}\right|$

In the sensor frame, consider two points on the $x y$ plane: $(x, y)$ and $(x+d x, y+d y)$. The Euclidean distance between them is $\Delta d=\sqrt{d x^{2}+d y^{2}}$. We are interested in statistical variations in the absolute value of the difference in elevation between these two points: $\Delta z_{\Delta d}=\left|z_{x+d x, y+d y}-z_{x, y}\right|$. Yokoya's method requires the expected value (first moment) of the distribution of elevation differences.

Because the data points are distributed irregularly on the $x y$ plane in the sensor frame, we must extend the original methods, which assume the data is distributed regularly (i.e., that the sampling interval is constant). For $i=0,1, \ldots, m$, and $\Delta d_{i}<\Delta d_{i+1}$, we prepare counters $A_{i}$ and $C_{i}$ to correspond to distance $\Delta d_{i}$. Let $\xi$ be a small distance that satisfies $0<\xi<\Delta d_{i}$, for any $i$. This parameter represents the width of a circular permissible area including a circle of radius $\Delta d_{i}$ (Figure 4). Suppose there is a data point at $(x+d x, y+d y)$ with elevation $z^{\prime}$. If $\left|\Delta d_{i}-\Delta d\right|$ is less than $\xi$, then the point lies in the permissible area, and we update the counters as follows:

$$
A_{i} \leftarrow A_{i}+\left|z^{\prime}-z\right|, \quad C_{i} \leftarrow C_{i}+1 .
$$




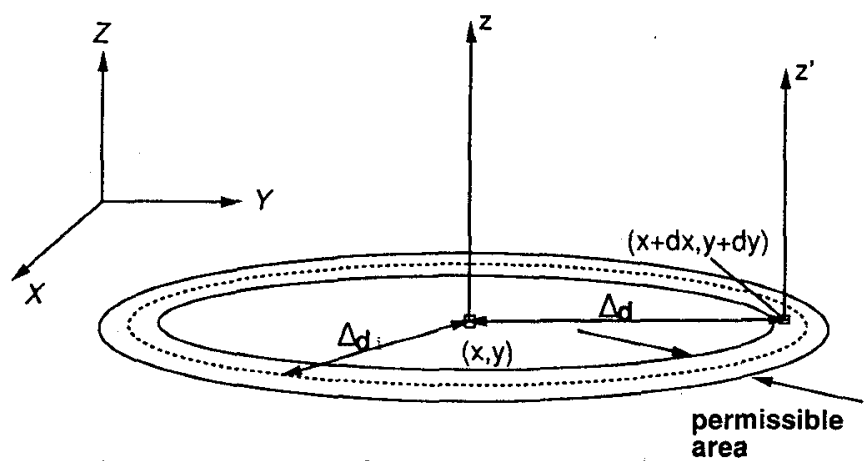

Figure 4: Accommodating irregular sampling intervals Because of irregular sampling, we cannot detect a pair of points located a certain distance $\Delta d_{i}$ away on the $x y$ plane. As a permissible area, we set a circle whose width is $2 \xi$ around a point $(x, y)$ with $z$. If the point $(x+d x, y+d y)$ exists in the area, its elevation $z^{\prime}$ is used to compute $A_{i}$

After considering all pairs of data points, we ensure that $C_{i}$ is larger than a threshold on the number of pairs. If $C_{i}$ is small, then we question whether the number of samples was sufficient to compute reliable statistics, and discard this data. Otherwise, we compute the sample mean by

$$
E_{\Delta d_{i}}=E\left[\mid z_{x+d x, y+d y}-z_{x, y} \|=A_{i} / C_{i}\right.
$$

4. Plot the points in log-log space and identify linear segments.

The point coordinates are $\left(\log \Delta d_{i}, \log E_{\Delta d_{i}}\right)$. Because most natural patterns exhibit self-similarity only over certain scales, and not over all scales, it is necessary to segment sets of points that are linear. We applied polyline fitting using the minimax method, as proposed by Kurozumi [6], to this segmentation problem. In the field of document image processing, this technique is frequently used to detect linear segments. The technique segments the given points into several sets of points which distribute within narrow rectangles, i.e., nearly along lines. The width of the rectangle must be specified as a threshold. In general, as the result of polyline fitting, we acquire several sets of points which distribute along lines. Among the sets that distribute along a line whose slope is between 0 and 1 , we choose the one whose extension along $\log \Delta d$ is the longest.

5. Estimate fractal dimension from the slope of a linear segment.

When the points lie on a line in the $\log$-log space, we can estimate the fractal dimension of the pattern by the difference between the Euclidean dimension of the pattern and the slope of the line formed by the points. The line is calculated using least-square fitting, and the fitting error may be regarded as a measure of the "fractalness" of the pattern.

\section{Experiments}

We selected eight patterns from range images acquired with the Perceptron rangefinder. Figure 5 illustrates some of the images.

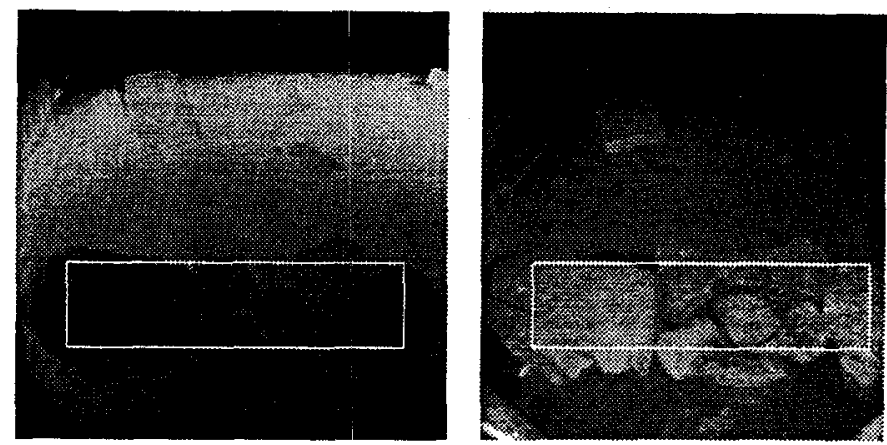

Pattern 2

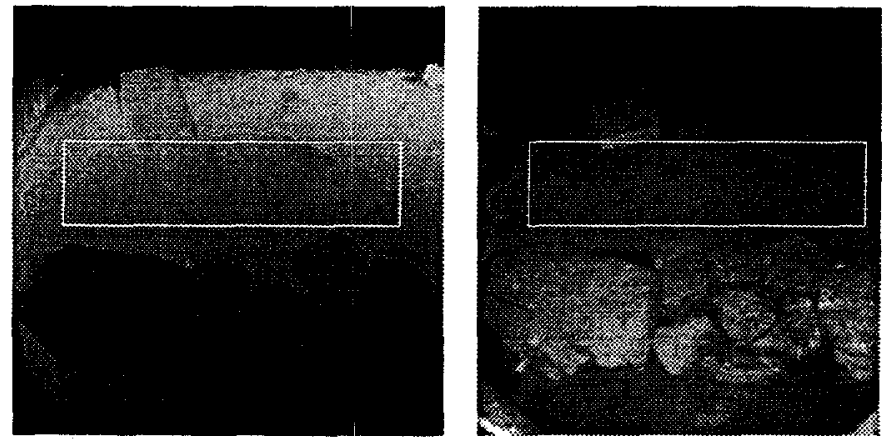

Pattern 4

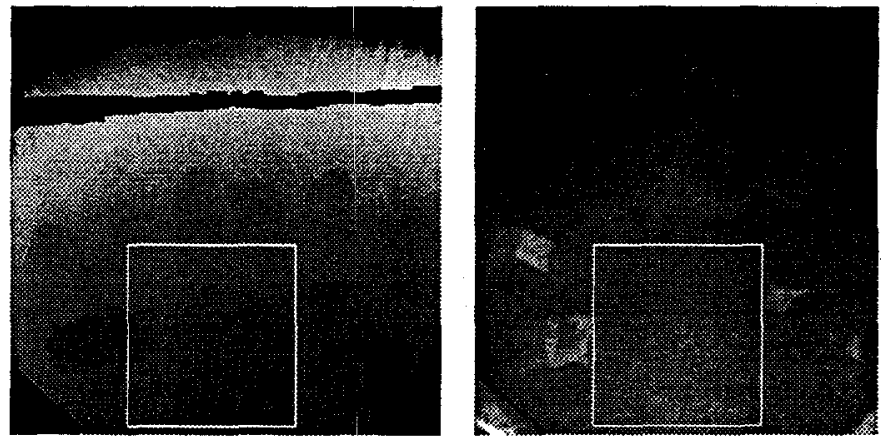

Pattern 6

Figure 5: Rangefinder images

The figure illustrates Perceptron image pairs: processed range (left) and raw reflectance. Only the range images are used to estimate fractal dimension.

The pattern numbers are presented in order of decreasing roughness, as determined subjectively by naive observers. We estimated the fractal dimension of the regions indicated by white rectangles.

Before applying the procedures to estimate fractal dimension, we checked whether the data acquired by the Perceptron satisfies the conditions on fractal Erownian functions stated in (1). Figure 6 histograms $z_{x+d x, y+d y}-z_{x, y}$ for Pattern 2, with $\Delta d=0.4,0.6$ and $0.8 \mathrm{~m}$. ¿From the figure it is clear that the condition in (1), that $g(x)$ be a cumulative distribution function, is satisficd. We conclude that it is not unreasonable to consider the patterns to be fractal Brownian functions.

A further condition on the distributions, imposed by Yokoya's method, is that they are normal. We conducted $\chi^{2}$ tests for Gaussianity and observed negative results, i.e., that the probabilities of the data being normally distributed were low. This suggests that it is not probable that the points were created by a 

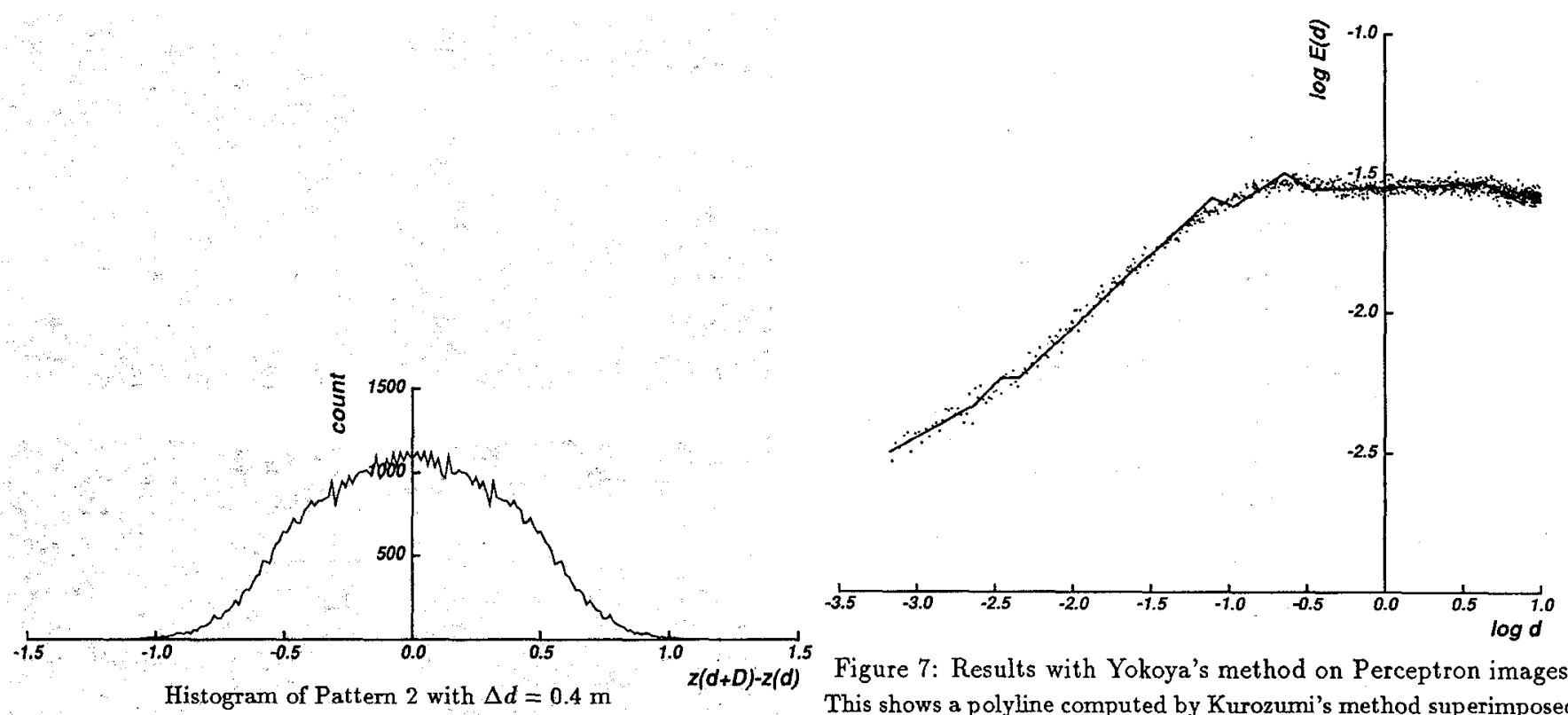

Figure 7: Results with Yokoya's method on Perceptron images This shows a polyline computed by Kurozumi's method superimposed on the points $\left(\log \Delta d, \log E_{\Delta d}\right)$ computed from pattern 2 .

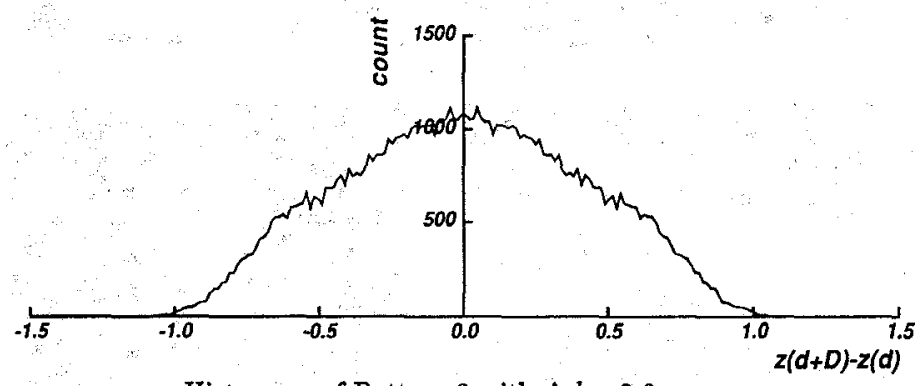

fractional Brownian motion process (which is a special case of a fractal Brownian function). However, to the extent that the distributions are symmetric, and exhibit a central tendency, there is some justification in proceeding to apply Yokoya's method, despite the negative $\chi^{2}$ test results.

Figure 7 illustrates the result of polyline fitting to a set of the points $\left(\log \Delta d, \log E_{\Delta d}\right)$ computed from range data named

Histogram of Pattern 2 with $\Delta d=0.6 \mathrm{~m}$ pattern 2. The set of plotted points consists of several subsets of points which distribute along lines. We set the width of the rectangle for polyline fitting to be 0.05 .

Figure 8 shows the result of applying the method described

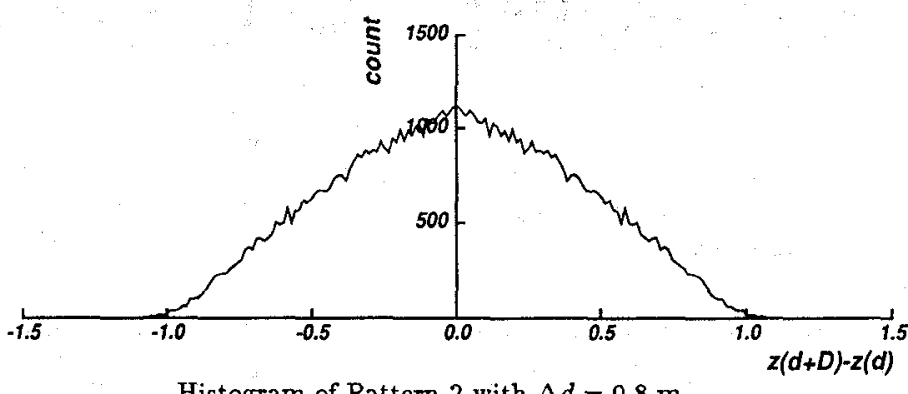
in the previous section: the linear segment of points segmented by polyline fitting and selected. In some ranges of scale, the points distribute almost along lines whose slopes are between 0 and 1 , therefore, we observe fractalness in all of these natural terrain patterns. For all the experiments, we set the width of the permissible area $\xi$ at $2 \times 10^{-4}$ meters and the threshold on a counter $C_{i}$ at 2000 pairs of points.

Table 1 lists the fractal dimensions estimated by the method. We also illustrate the fitting error normalized by $\log E\left(\Delta z_{\Delta d}\right)$.

Figure 6: Empirical distribution functions These graphs illustrate histograms of $z_{x, y}-z_{x+d x, y+d y}$ with $\Delta d=$ $\sqrt{d x^{2}+d y^{2}}$ on Pattern 2 .
All the errors are small enough to determine that the patterns are fractals. Moreover, the rows are ordered by roughness, as perceived by naive observers. The order of estimated fractal dimension correlate strongly to the intuitive order (the last three patterns-sandy flat floors-are almost identical). These results suggest that the fractal dimension estimated using these methods can be utilized as a measure of roughness of natural terrain.

Expanding the method, we can estimate dense distribution of fractal dimension from range images. The fractal dimension of each pixel is estimated from a rectangular window around the interest pixel. Figure 9 illustrates an experimental result on a part of Pattern 2. We set the window size at 40 pixels. The range of the estimated fractal dimension is between 2.1 and 2.7. In the resulted image, the brighter is the pixel, the higher is the 

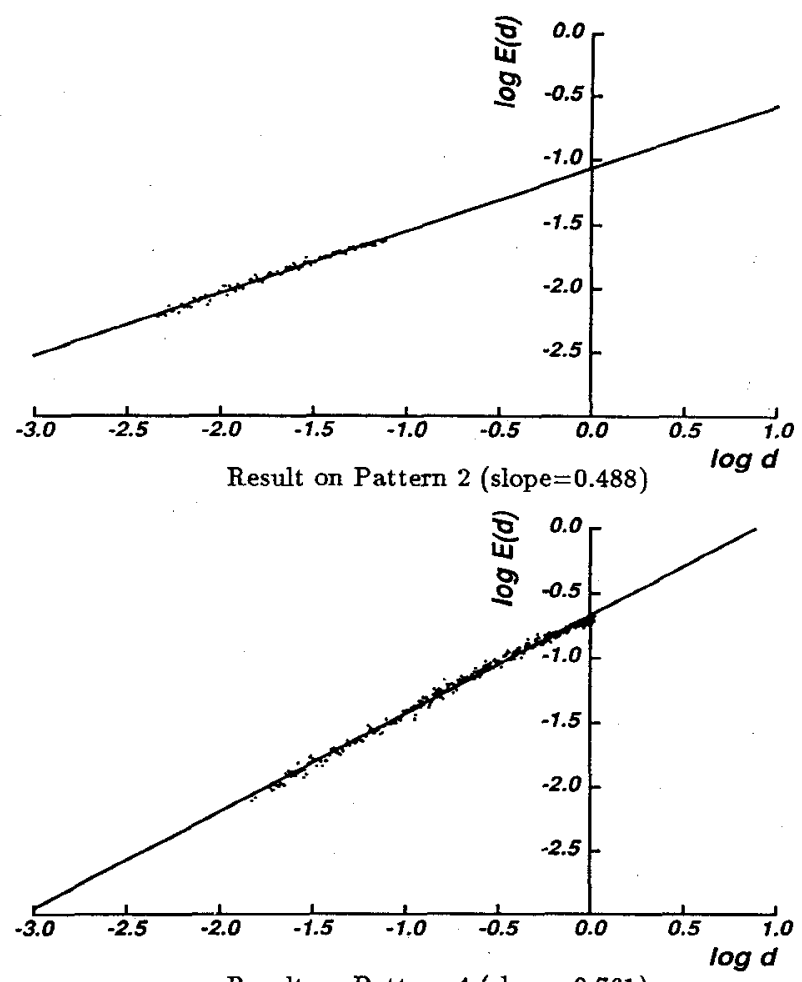

Result on Pattern 4 (slope $=0.761$ )

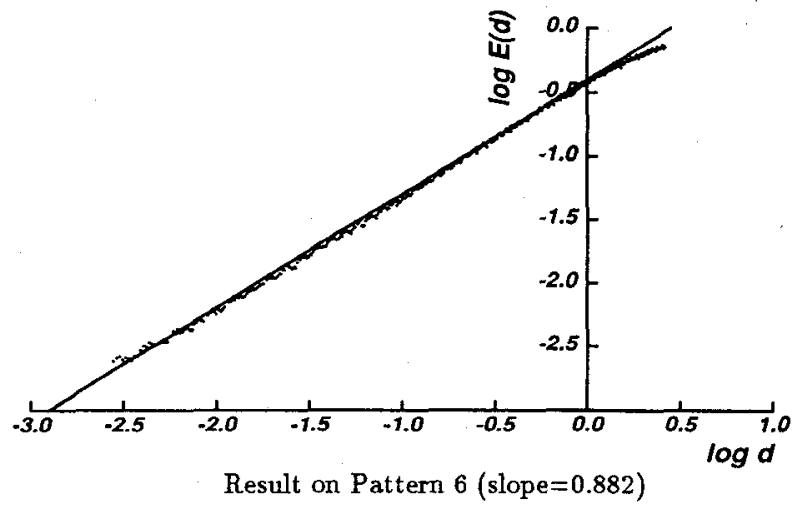

Figure 8: Results with Yokoya's method on Perceptron images These graphs illustrate the segmented points and the lines fitted to them. The label $(\log d, \log E(d))$ corresponds to $\left(\log \Delta d, \log E_{\Delta d}\right)$ in the text.

\begin{tabular}{c|cc} 
Pattern & fractal dimension (error) \\
\hline 1 & 2.661 & $(0.025)$ \\
2 & 2.512 & $(0.016)$ \\
3 & 2.486 & $(0.014)$ \\
4 & 2.239 & $(0.025)$ \\
5 & 2.196 & $(0.010)$ \\
6 & 2.118 & $(0.021)$ \\
7 & 2.102 & $(0.014)$ \\
8 & 2.010 & $(0.022)$
\end{tabular}

Table 1: Fractal dimensions estimated by the proposed method
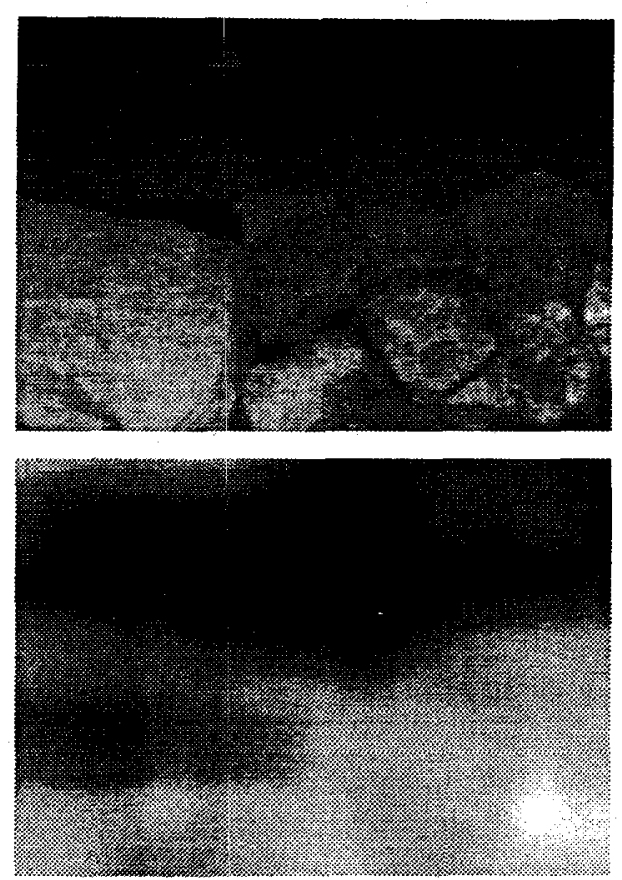

Figure 9: Dense estimation of fractal dimension from a range image

The top image is the intensity image corresponding to the original range image. The bottom image is dense distribution of estimated fractal dimension. The brighter is the pixels, the higher is the estimation.

fractal dimension. We can find the edges of rocks even in the resulting image. This is applicable to, for example, gait planning for legged robots. Then we should avoid putting the leg to the bright area.

The computational complexity of the method utilized here is $O\left(N^{2}\right)$, where $N$ is the number of pixels, because it is necessary to calculate distances between all pairs of pixels in order to determine which pairs lie in the permissible area. On a Sun $4 / 40$ with $24 \mathrm{MB}$ of physical memory, estimating the fractal dimension shown in Table 1 for Pattern 2 (10000 pixels) requires $1.2 \times 10^{3}$ sec. A parallel version of the algorithm runs 330 times faster (for $10^{18}$ data points) on a Maspar MP1 with 4096 processors [8].

\section{Conclusion}

The roughness of a shape is an ambiguous property. Geometrically, the Hausdorff-Besicovich dimension is a measure of elasticity, which is one of the main factors contributing to the notion of roughness [4]. The Hausdorff-Besicovich dimension is defined on general geometrical shapes, but it is equal to the fractal dimension only on fractals. Natural terrain is typically a fractal (over some range of scales). Therefore, its fractal dimension can be used to express its roughness properly.

In this paper, we concentrated on the fractal Brownian function approach for estimating fractal dimension. We extended the fractal Brownian function approach to accommodate irregularly sampled data supplied by a scanning laser rangefinder. The extension involves considering neighbors that lie approximately, 
but not exactly, within a given distance from a data point. This requires searching a permissible area within the given distance. The extension also involves analysis of the distribution of points in a log-log space in order to determine which points to analyze further and which points to ignore (most real patterns are fractal only over some range of scales, not at all scales). We conduct this analysis by applying a segmentation method using polyline fitting to the points in log-log space.

We applied the extended methods to noisy range imagery of natural terrain (sand and rocks) acquired with the Perceptron scanning laser rangefinder located on a prototype of a planetary rover. The resulting estimates of fractal dimension correlate closely to the human perception of the roughness of the terrain. We conclude that it is reasonable to model natural terrain as a fractal pattern, and that the fractal dimension is a reasonable measure of roughness of terrain.

Remaining problems in this work that we do not intend to pursue immediately include determining the region in which to conduct fractal analysis, identifying which linear parts of the loglog curves are most significant, and segmenting patterns whose fractal characteristics change spatially.

\section{References}

[1] K. Arakawa and E. Krotkov. Estimating Fractal Dimension from Range Images of Natural Terrain. Technical Report CMU-CS-91-156, School of Computer Science, Carnegie Mellon University, Pittsburgh, Pennsylvania, July 1991.

[2] K. Arakawa and E. Krotkov. Fractal Surface Reconstruction with Uncertainty Estimation: Modeling Natural Terrain. Technical Report CMU-CS-92-1194, School of Computer Science, Carnegie Mellon University, Pittsburgh, Pennsylvania, October 1992.

[3] J. Bares, M. Hebert, T. Kanade, E. Krotkov, T. Mitchell, R. Simmons, and W. Whittaker. Ambler: An Autonomous Rover for Planetary Exploration. IEEE Computer, 22(6):18-26, June 1989.

[4] H. Kaneko. Fractal and Its Application to Image Analysis. Journal of the Institute of Television Engineers of Japan, $41(4): 359-366,1987$. In Japanese.

[5] P. Kube and A. Pentland. On the Imaging of Fractal Surfaces. IEEE Trans. on Pattern Analysis and Machine Intelligence, PAMI-10(5):704-707, September 1988.

[6] Y. Kurozumi and W. Davis. Polygonal Approximation by the Minimax Method. Computer Graphics and Image Processing, 19:248-264, 1982.

[7] I. Kweon, R. Hoffman, and E. Krotkov. Experimental Characterization of the Perceptron Laser Rangefinder. Technical Report CMU-RI-TR-91-1, Robotics Institute, Carnegie Mellon University, Pittsburgh, Pennsylvania, January 1991.
[8] L.-K. Lim. Algorithm for Estimating Fractal Dimension on a Massively Parallel Computer. Senior thesis in Mathematics and Computer Science, Carnegie Mellon University, May 1993.

[9] B. Mandelbrot. The Fractal Geometry of Nature. Freeman, San Francisco, California, 1982.

[10] A. Pentland. Fractal-Based Description of Natural Scenes. IEEE Trans. on Pattern Analysis and Machine Intelligence, 6(6):661-674, 1984.

[11] R. Szeliski. Baysian Modeling of Uncertainty in Low-Level Vision. $\mathrm{PhD}$ thesis, Carnegie Mellon University, August 1988. Available as Technical Report CMU-CS-88-169.

[12] R. Voss. Random Fractal Forgeries. In R. Earnshaw, editor, Fundamental Algorithms for Computer Graphics, pages 805835 , Berlin, 1985. Springer-Verlag.

[13] R. Voss. Fractals in Nature: Characterization, Measurement, and Simulation. In H.-O. Peitgen and D. Saupe, editors, The Science of Fractal Images. Springer-Verlag, 1988.

[14] N. Yokoya. Fractal Based Analysis of 3D Complex Shapes and Its Applications. Technical Report PRU86-23, IECEJ, 1986. In Japanese.

[15] N. Yokoya, K. Yamamoto, and N. Funakubo. Fractal-Based Analysis and Interpolation of 3D Natural Surface Shapes and Their Application to Terrain Modeling. Computer Vision, Graphics, and Image Processing, 46(3):284-302, June 1989. 